\title{
A novel modified Omega-K algorithm for circular trajectory scanning SAR imaging using series reversion
}

\author{
Yi Liao*, Meng-dao Xing, Lei Zhang and Zheng Bao
}

\begin{abstract}
This article presents a modified Omega-K algorithm for circular trajectory scanning synthetic aperture radar (CTSSAR) imaging. Due to the curvature of circular trajectory, it is difficult to have access to the two-dimensional frequency spectrum for CTSSAR via the principle of stationary phase (POSP), as conventional SAR imaging methods $\mathrm{RD}$ and CS. Herein, the analytic point target spectrum is first derived by series reversion and the POSP, based on which a modified Omega-K algorithm is developed to focus data accurately. The accuracy can be controlled by keeping enough terms in the two series expansions so that a well-focused image can be achieved with a proper range approximation. After detailed analyses and experiments, the fourth-order approximation is proved to be the best choice. Furthermore, the computational efficiency is evaluated by comparing the given method with the back projection algorithm and other methods with different approximated orders. The proposed algorithm is verified to be the best one in terms of computational burden. A well-focused image is obtained by simulations, validating the feasibility of the proposed algorithm.
\end{abstract}

Keywords: Circular trajectory scanning (CTS) synthetic aperture radar (SAR) (CTSSAR), Series reversion, Omega-K algorithm

\section{Introduction}

Due to its capability of working day/night and all weather conditions, synthetic aperture radar (SAR) has widely been applied to the military and civilian practical uses. The traditional SAR system often operates along a straight path at a certain altitude with respect to the ground plane [1-4]. In recent years, the curved path SAR has attracted more and more attention of many researchers. An imaging mode called circular SAR (CSAR), whose radar system moves along a circular trajectory, gradually became one of the hotspots in the field of radar signal processing [5]. In CSAR imaging mode, the sensor steers its antenna beam to illuminate a certain terrain patch, like "circular spotlight" mode to some extent. CSAR owns many advantages over the conventional one that it has the capability to achieve subwavelength resolution in the ground plane due to its aperture of $360^{\circ}$. What is more, the multi-aspect

* Correspondence: fjsdfzly@126.com

National Key lab of Radar Signal Processing, Xidian University, Xi'an 710071, P.R. China observation of CSAR provides the information of the interested target from different azimuth directions, making it possible to realize a $3 \mathrm{D}$ reconstruction [6-14]. Although CSAR owns so many advantages, the imaging area is relatively small. Usually, both the height and the diameter of the imaging area in the ground plane are several hundred meters [15]. Therefore, it is suitable for the high-resolution imaging of an interested area.

A new wide area circular trajectory scanning SAR (CTSSAR) imaging mode has raised the interests of a growing numbers of researchers [16]. In this mode, the platform moves along a circular path at a certain altitude with its antenna beam pointing fixed perpendicular to the flight velocity away from the center of the circular trajectory, the result is a moving antenna footprint that sweeps along an annular terrain. Just like the spotlight SAR and CSAR, the only thing differs CTSSAR from the conventional stripmap is the shape of the track. Hence, we prefer to call CTSSAR as 'circular stripmap' similarly. Although certain sacrifices in azimuth resolution may occur, CTSSAR can possess a larger imaging area than

\section{穴}


that of the traditional stripmap mode for its higher scanning speed in azimuth, so it is more suitable for fast wide area imaging.

Due to the trajectory curvature of CTSSAR, there are trigonometric functions under the radical sign in the target's range function. The accurate analytical expression of the 2D spectrum is not achievable by adopting conventional principle of stationary phase, which becomes an obstacle in the way of designing a fast frequency- domain-based algorithms for CTSSAR imaging. Thus, Sun et al. [16] employ a quadratic approximation to the range function in its deduction of the spectrum to design an imaging method. In the case that the synthetic aperture is relatively short and the azimuth resolution is low, the impact of the curved trajectory can be neglected, and satisfactory results can be reached using this method. Nevertheless, the quadratic approximation method ignores the high-order range terms. Under the condition that the
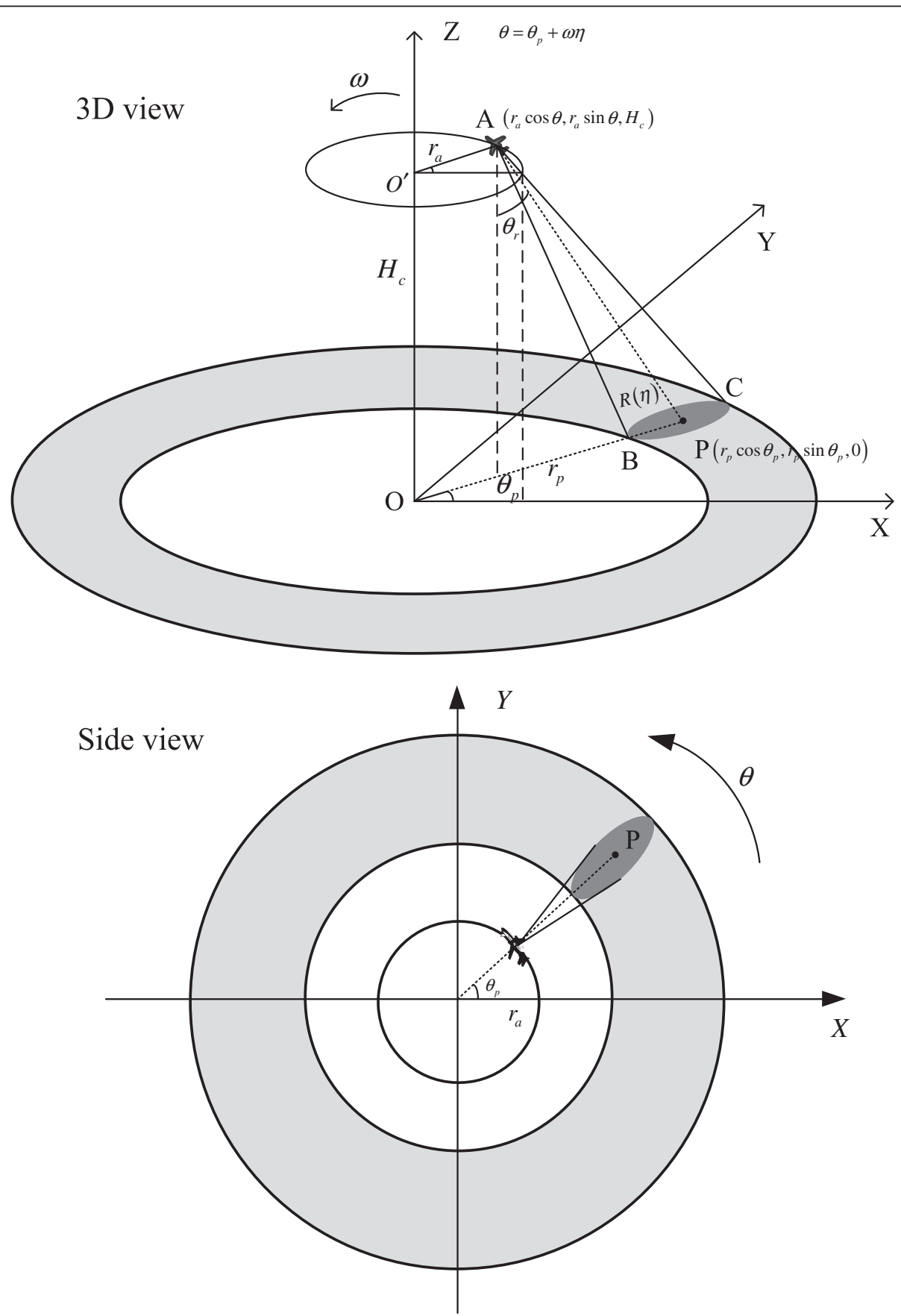

Figure 1 Imaging geometry of the CTSSAR. 
integration time is long and the azimuth resolution is high, the range errors introduced by this approximation will be large enough to defocus the image. The back projection (BP) algorithm shown in [10] is a classical time-domain algorithm, which can get rid of the problem of spectrum derivation in the frequency-domain-based algorithms and be implemented easily with arbitrary geometries. But the disadvantage is that each pixel must be compensated individually, leading to a heavy computational burden.

In order to obtain a more applicable approach to focus CTSSAR data, a novel imaging algorithm for CTSSAR is proposed based on the method of series reversion (MSR). The MSR is a method using the Doppler series expansion coefficients to obtain the coefficients of the stationary phase by reversion $[17,18]$. The accuracy of the spectrum is controlled by keeping enough terms in the two series expansions. Moreover, the corresponding modified Omega- $\mathrm{K}$ algorithm is established based on the derived 2D spectrum.

In this article, the imaging geometry model is analyzed first and the involved problem is discussed as well. To obtain the 2D spectrum based on MSR, the range function is kept up to the fourth-order. Thus, a new modified Omega-K algorithm tailored for CTSSAR over a circular path is developed and the detailed flowchart is given. Finally, the algorithm is tested with satisfying results by simulations for point targets in different range. Implementation aspects, including resolutions, computational complexity, and the sensitivity to motion errors, are also assessed in this article.

\section{Signal model for CTSSAR}

The imaging geometry for CTSSAR is shown in Figure 1. The radar platform moves along a circular path of radius $r_{a}$ with height $H_{c}$ on a plane parallel to the ground plane. As the radar moves, its beam is in the plane perpendicular to the flight velocity all the time. When the radar platform moves around a whole circle centered at the origin point $\mathrm{O}$ of the spatial coordinate, the beam-illuminating scene will be an annular area with inner radius $\mathrm{OB}$ and outer radius $\mathrm{OC}$. The coordinate of radar platform $\mathrm{A}$ is denoted by $\left(r_{a} \cos \theta, r_{a} \sin \theta, H_{c}\right)$, where the aspect angle is $\theta$. Assume that there is an arbitrary point target $P$ located at $\left(r_{p} \cos \theta_{p}, r_{p} \sin \theta_{p}, 0\right)$. For simplicity of the following derivation, let us define that the slow time is zero when $\theta=\theta_{p}$, namely, the beam center crossing time. The angle velocity is denoted by $\omega$, and the slow time is represented by $\eta$. So, we have $\theta=\theta_{p}+\omega \eta$. Thus, the instantaneous range $R(\eta)$ between the radar and the target $P$ can be obtained based on the law of cosines as shown in the following expressions.

$$
H_{c} \tan \theta_{r}=r_{p}-r_{a}
$$

$$
\begin{aligned}
R(\eta) & =\sqrt{H_{c}^{2}+r_{p}^{2}+r_{a}^{2}-2 r_{a} r_{p} \cos }\left(\theta-\theta_{p}\right) \\
& =\sqrt{H_{c}^{2}+r_{p}^{2}+r_{a}^{2}-2 r_{a} r_{p} \cos \omega \eta}
\end{aligned}
$$

Defining $R_{\text {cen }}=\sqrt{H_{c}^{2}+\left(r_{p}-r_{a}\right)^{2}}$, we perform Taylor expansion to the instantaneous range neglecting the terms whose order are higher than the fourth order. The expression can be written as

$$
R(\eta)=R_{\mathrm{cen}}+k_{1} \eta+k_{2} \eta^{2}+k_{3} \eta^{3}+k_{4} \eta^{4}+\mathrm{K}
$$

where

$$
\begin{aligned}
k_{1} & =0, k_{2}=\frac{r_{a} r_{p} \omega^{2}}{2 R_{\mathrm{cen}}}, k_{3}=0, k_{4} \\
& =-\frac{\omega^{4} r_{a} r_{p}}{24 R_{\mathrm{cen}}}-\frac{\omega^{4} r_{a}^{2} r_{p}^{2}}{8 R_{\mathrm{cen}}^{3}}
\end{aligned}
$$

Assume that the transmitting radar signal is linear frequency modulation signal, the pulse width is $T_{\mathrm{p}}$, and the rate of frequency modulation is $\gamma$. The echo from $P$ can be presented by

$$
\begin{aligned}
s_{r}(t, \eta)= & \sigma_{p} a_{r}\left[t-\frac{2 R(\eta)}{c}\right] a_{a}(\eta) \cdot \exp \left\{j \pi \gamma\left[t-\frac{2 R(\eta)}{c}\right]^{2}\right\} \cdot \exp \\
& \times\left[-j \frac{4 \pi}{\lambda} R(\eta)\right]
\end{aligned}
$$

where $\sigma_{p}$ is the coefficient of reflectivity, $a_{r}(\bullet)$ and $a_{a}(\bullet)$ are the range envelope and azimuth envelope, respectively, $c$ is the speed of light, and $\lambda$ is the wavelength according to the center frequency.

Now, convert the echo signal shown in (5) to the range frequency azimuth time domain,

$$
\begin{aligned}
S_{0}\left(f_{\tau}, \eta\right)= & \sigma_{p} A_{r}\left(f_{\tau}\right) a_{a}(\eta) \cdot \exp \left[-j \frac{4 \pi\left(f_{c}+f_{\tau}\right) R(\eta)}{c}\right] \cdot \exp \\
& \times\left(-j \frac{\pi f_{\tau}^{2}}{\gamma}\right)
\end{aligned}
$$

where $f_{c}$ is the carrier frequency, $f_{\tau}$ is the range frequency, and $A_{r}\left(f_{\tau}\right)$ represents the envelope of the range frequency. Now let us try to figure out the 2D spectrum using the stationary phase method as follow, first azimuth FFT is performed to (6), yielding

$$
\begin{aligned}
S_{2}\left(f_{\tau}, f_{\eta}\right) & =\int_{-\infty}^{+\infty} S_{0}\left(f_{\tau}, \eta\right) \exp \left(-\mathrm{j} 2 \pi f_{\eta} \eta\right) \mathrm{d} \eta \\
& =\int_{-\infty}^{+\infty} \sigma_{p} A_{r}\left(f_{\tau}\right) a_{a}(\eta) \cdot \exp \{\theta(\eta)\} \mathrm{d} \eta
\end{aligned}
$$


The phase in the Fourier integral is

$$
\theta(\eta)=-\frac{4 \pi\left(f_{c}+f_{\tau}\right) R(\eta)}{c}-\frac{\pi f_{\tau}^{2}}{\gamma}-2 \pi f_{\eta} \eta
$$

Then operate on the derivatives of $\theta(\eta)$ with respect to the components of $\eta$,

$$
\begin{aligned}
\frac{d \theta(\eta)}{d \eta}= & -\frac{4 \pi\left(f_{c}+f_{\tau}\right)}{c} \frac{\omega r_{a} r_{p} \sin \omega \eta}{\sqrt{r_{a}^{2}+H_{c}^{2}+r_{p}^{2}-2 r_{a} r_{p} \cos \omega \eta}} \\
& -2 \pi f_{\eta}
\end{aligned}
$$

Let it be zero, yielding

$$
f_{\eta}=-\frac{2\left(f_{c}+f_{\tau}\right) \omega r_{a} r_{p} \sin \omega \eta}{c \sqrt{r_{a}^{2}+H_{c}^{2}+r_{p}^{2}-2 r_{a} r_{p} \cos \omega \eta}}
$$

From (9), it can be noticed that there are trigonometric functions in both numerator and denominator of the azimuth Doppler frequency, which will make the deduction of $\eta$ by (9) very hard to carry out and further put a huge obstacle in the way of deriving the accurate $2 \mathrm{D}$ spectrum. This problem is caused by the curvature of the circular trajectory in CTSSAR. Thus, enough approximation is required to derive the $2 \mathrm{D}$ frequency spectrum and ensure a satisfied focusing result. Sun et al. [16] proposed a quadratic approximation method to make the spectrum expression similar to that of the traditional straight line stripmap mode. This approach ignores the curvature of the circular trajectory and the parabolic approximation is introduced, making the spectrum derivation relatively easy to implement. However, the precision of the obtained spectrum is relatively lower. In case of low-resolution and short integration time, i.e., when the impact of the curvature of the motion trajectory to the range variation can be neglected, the flight path for the target point can be regarded as straight line approximately. Thus, the method in [16] is suitable under this situation. Nevertheless, if the integration time is relatively long and the required azimuth resolution is high, the image might show significant degradation by adopting the original quadratic approximation method for its neglecting of the high-order terms. So, it is necessary to find a new spectrum deducing method for CTSSAR.

\section{2D spectrum and imaging algorithm}

\subsection{The deduction of the $2 \mathrm{D}$ spectrum based on MSR}

From the above analysis, it is obvious that due to the complicated range expression in the CTSSAR where the simple quadratic range equation does not hold, the stationary phase point is difficult to approach and the 2D spectrum is hard to obtain. Thus, MSR is adopted to deduce the $2 \mathrm{D}$ spectrum, and the stationary phase point's series expansion coefficients with respect to azimuth time will be estimated by reversing the coefficients in the Doppler spectrum, and the high accuracy $2 \mathrm{D}$ spectrum will be obtained. In the procedure of deduction, the accuracy of the deduced spectrum can be controlled by choosing an approximating order in the expansion, which will be discussed in detail in Section 4. In addition, the effective algorithm for CTSSAR will be designed based on the spectrum.

As for (9), let the derivative be zero [16], and the Doppler frequency can be written as follows:

$$
\left(-\frac{c}{f_{c}+f_{\tau}}\right) f_{\eta}=2 k_{2} \eta+3 k_{3} \eta^{2}+4 k_{4} \eta^{3}+\mathrm{L}
$$

Using MSR, we can get the azimuth time in the form of Doppler frequency expansion, i.e., the expression of the stationary phase point which is presented as follows:

$$
\begin{aligned}
\eta\left(f_{\eta}\right)= & A_{1}\left(-\frac{c}{f_{c}+f_{\tau}} f_{\eta}\right)+A_{2}\left(-\frac{c}{f_{c}+f_{\tau}} f_{\eta}\right)^{2} \\
& +A_{3}\left(-\frac{c}{f_{c}+f_{\tau}} f_{\eta}\right)^{3}+\mathrm{L}
\end{aligned}
$$

where

$$
\begin{aligned}
A_{1} & =\frac{1}{2 k_{2}}=\frac{R_{c e n}}{\omega^{2} r_{a} r_{p}}, A_{2}=0, A_{3} \\
& =\frac{-1}{\left(2 k_{2}\right)^{5}} \cdot 2 k_{2} \cdot 4, k_{4}=\frac{R_{c e n}^{3}}{6 \omega^{4} r_{a}^{3} r_{p}^{3}}+\frac{R_{c e n}}{2 \omega^{4} r_{a}^{2} r_{p}^{2}}
\end{aligned}
$$

According to (6) and (12), it is easy to get the following equation

$$
\begin{gathered}
S_{A}\left(f_{\tau}, f_{\eta}\right)=\sigma_{p} A_{r}\left(f_{\tau}\right) A_{a}\left(f_{\eta}\right) \exp \left\{-j 2 \pi f_{\eta} \eta\left(f_{\eta}\right)\right\} \\
\cdot \exp \left\{-j \frac{4 \pi\left(f_{c}+f_{\tau}\right)}{c} R\left(\eta\left(f_{\eta}\right)\right)-j \frac{\pi f_{\tau}^{2}}{\gamma}\right\}
\end{gathered}
$$

where $A_{a}\left(f_{\eta}\right)$ is the envelope of the Doppler frequency. What should be pointed out here is that the odd terms are zero due to the trigonometric functions in the range equation. It is necessary to keep (14) to a certain order when the image is well focused. The analysis of it will be mentioned in detail in Section 4. Moreover, according to (12), (13) and (14), the phase in the 2D spectrum of the target point signal can be rewritten as follows:

$$
S_{2 d f}\left(f_{\tau}, f_{\eta}\right)=\sigma_{p} A_{r}\left(f_{\tau}\right) A_{a}\left(f_{\eta}\right) \exp \left(j \varphi\left(f_{\tau}, f_{\eta}\right)\right)
$$

and the phase term in (15) can be shown as follows:

$$
\begin{aligned}
\varphi\left(f_{\tau}, f_{\eta}\right)= & -\frac{4 \pi\left(f_{c}+f_{\tau}\right)}{c} R_{\mathrm{cen}}+\frac{\pi}{k_{2}} \frac{c}{4\left(f_{c}+f_{\tau}\right)} f_{\eta}^{2} \\
& -\frac{\pi k_{4}}{64 k_{2}^{4}}\left(\frac{c}{f_{c}+f_{\tau}}\right)^{3} f_{\eta}^{4}-\frac{\pi f_{\tau}^{2}}{\gamma}
\end{aligned}
$$


In (16), the phase in the 2D spectrum is kept up to the fourth-order with respect to $f_{\eta}$, and a more precise spectrum is obtained. Herein, the third term on the right-hand side of the equal sign indicates the curvature of the circular trajectory. In the following section, the imaging algorithm for CTSSAR will be given based on the $2 \mathrm{D}$ spectrum derived by the above analysis.

\subsection{Imaging algorithm}

Our objective is to obtain the frequency-domain signal containing the linear terms of the target only. It can be seen from the above deduction that there are variables such as $f_{\tau}, f_{\eta}$, and $R(\eta)$ in the spectrum. Due to the existence of the coupling between the range frequency $f_{\tau}$ and the Doppler frequency $f_{\eta}$ in the $2 \mathrm{D}$ spectrum, it is not easy to get the separated linear frequency term. Herein, the Taylor series expansion of (16) is operated in $f_{\tau}=0$, i.e.,

$$
\begin{aligned}
\theta_{c}= & -\frac{4 \pi\left(f_{c}+f_{\tau}\right) R_{\mathrm{cen}}}{c}+\varphi_{a z}\left(f_{\eta}\right)+\varphi_{\mathrm{rcm}}\left(f_{\eta}\right) f_{\tau} \\
& +\varphi_{\mathrm{src}}\left(f_{\eta}\right) f_{\tau}^{2}+\varphi_{\mathrm{rg}}\left(f_{\tau}\right)+\varphi_{\mathrm{res}}\left(f_{\eta}, f_{\tau}\right)
\end{aligned}
$$

where $\frac{-4 \pi f_{\tau} R_{\mathrm{cen}}}{c}$ is the linear phase representing the location of target point, where the energy of the target can be focused within the corresponding range cell. $\frac{-4 \pi f_{c} R_{\text {cen }}}{c}$ is the constant term, which is independent from $f_{\tau}$ and $f_{\eta}$, so its influence to the focusing can be omitted. In addition, the other five terms can be shown as follows: a. Azimuth modulation term: The second phase term on the right-hand side of the equal sign of (17) is the azimuth modulation term, i.e.,

$$
\varphi_{\mathrm{az}}\left(f_{\eta}\right)=\frac{\pi \cdot c \cdot f_{\eta}^{2}}{4 k_{2} f_{c}}-\frac{\pi k_{4} c^{3} f_{\eta}^{4}}{64 k_{2}^{4} f_{c}^{3}}
$$

This term indicates the azimuth modulation. The azimuth modulation rate is range-variant for the fact that the $k$-coefficients are varied with range cells. The coupling between range and azimuth cannot be removed in the $2 \mathrm{D}$ frequency domain, so the term must be compensated in the range/Doppler domain. Herein, the azimuth compression is completed by multiplying the signal with the azimuth match filtering function after range cell migration (RCM) and range compression.

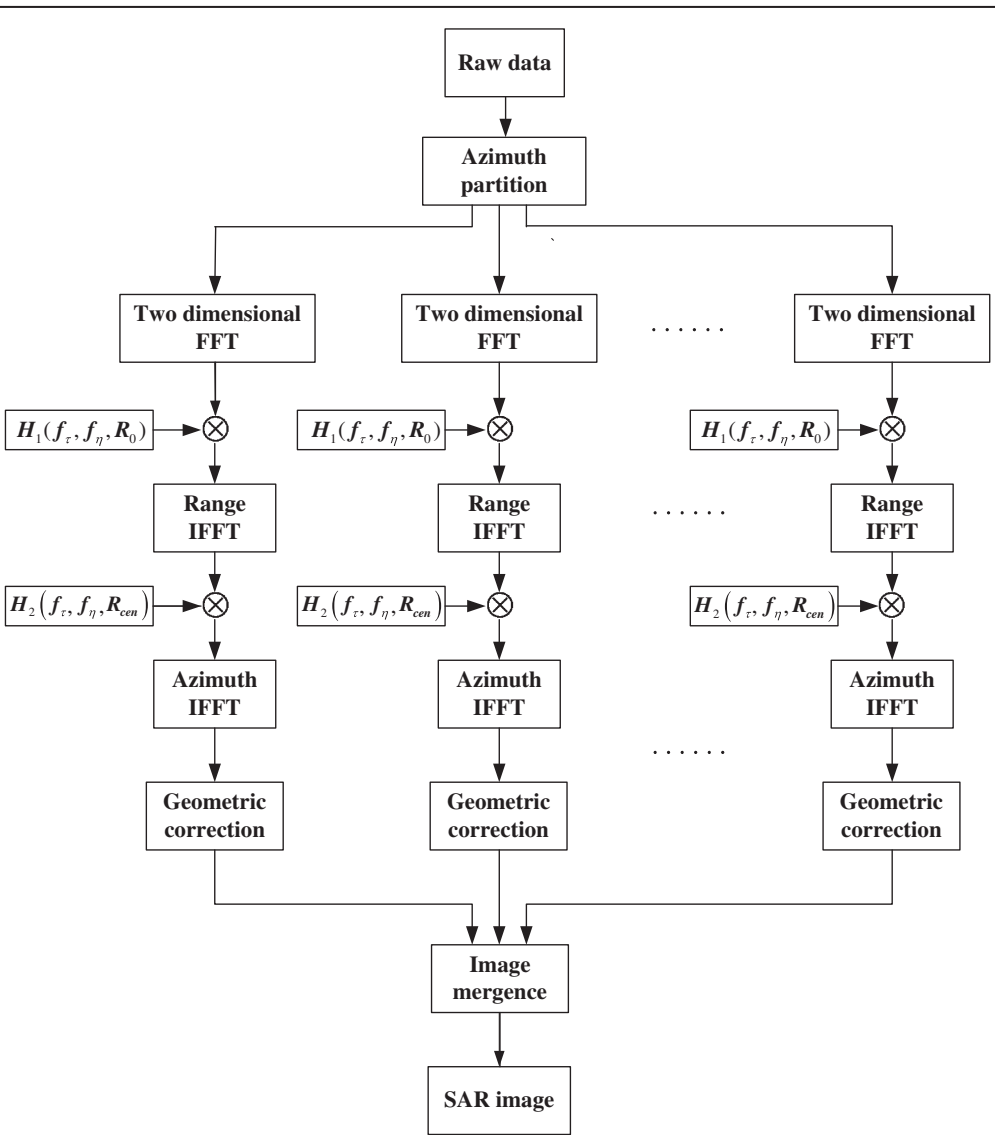

Figure 2 Full-scene SAR imaging algorithm flow chart. 
b. RCM term:

The third phase term on the right-hand side of the equal sign of (17) is the RCM term, i.e.,

$$
\varphi_{\mathrm{rcm}}\left(f_{\eta}\right)=-\frac{\pi \cdot c}{4 k_{2}}\left(\frac{f_{\eta}}{f_{c}}\right)^{2}+\frac{3 \pi k_{4} c^{3}}{64 k_{2}^{4}}\left(\frac{f_{\eta}}{f_{c}}\right)^{4}
$$

This term varies with $f_{\eta}$, representing the coupling between the range and the azimuth which needs to be compensated. For the common case of CTSSAR imaging, the range swath is not so wide that the RCM term's range variation can be neglected. In the following process, the scene center is chosen as the reference and the corresponding RCM correction function is established in the 2D frequency domain to realize the range migration correction.

c. Secondary range compression term (SRC):

The fourth phase term on the right-hand side of the equal sign of (17) is the SRC term, i.e.,

$$
\varphi_{\mathrm{src}}\left(f_{\eta}\right)=\frac{\pi \cdot C \cdot f_{\eta}^{2}}{4 k_{2} f_{c}^{3}}-\frac{3 \pi k_{4} c^{3} f_{\eta}^{4}}{32 k_{2}^{4} f_{c}^{5}}
$$

This term indicates the variation of the range modulation rate resulted from the coupling between $f_{\tau}$ and $f_{\eta}$. So, this term will impact the range focusing if not considered properly, while the variation of the SRC with range can be neglected usually. Thus, the compensated term in the $2 \mathrm{D}$ frequency domain is built with the scene center the reference to remove the influence of the SRC term to imaging.

d. Range modulation term:

The fifth phase term on the right-hand side of the equal sign of (17) is the range modulation term, i.e.,

$$
\varphi_{\mathrm{rg}}\left(f_{\tau}\right)=-\frac{\pi f_{\tau}^{2}}{\gamma}
$$

This term presents the range modulation of the transmitted signal, which is independent from $f_{\eta}$. It can be compensated in the $2 \mathrm{D}$ frequency domain as well.

e. Residue phase term:

The sixth phase term on the right-hand side of the equal sign of (17) is the residue phase term, i.e.,

$$
\begin{aligned}
\varphi_{\mathrm{res}}\left(f_{\eta}, f_{\tau}\right) & =-\frac{\pi \cdot c \cdot f_{\eta}^{2}}{4 k_{2}} \frac{f_{\tau}^{3}}{f_{c}^{3}\left(f_{c}+f_{\tau}\right)} \\
- & \frac{\pi k_{4} c^{3} f_{\eta}^{4}}{64 k_{2}^{4}}\left\{1 /\left(f_{c}+f_{\tau}\right)-1 / f_{c}^{3}+3 f_{\tau} / f_{c}^{4}-6 f_{\tau}^{2} / f_{c}^{5}\right\}
\end{aligned}
$$

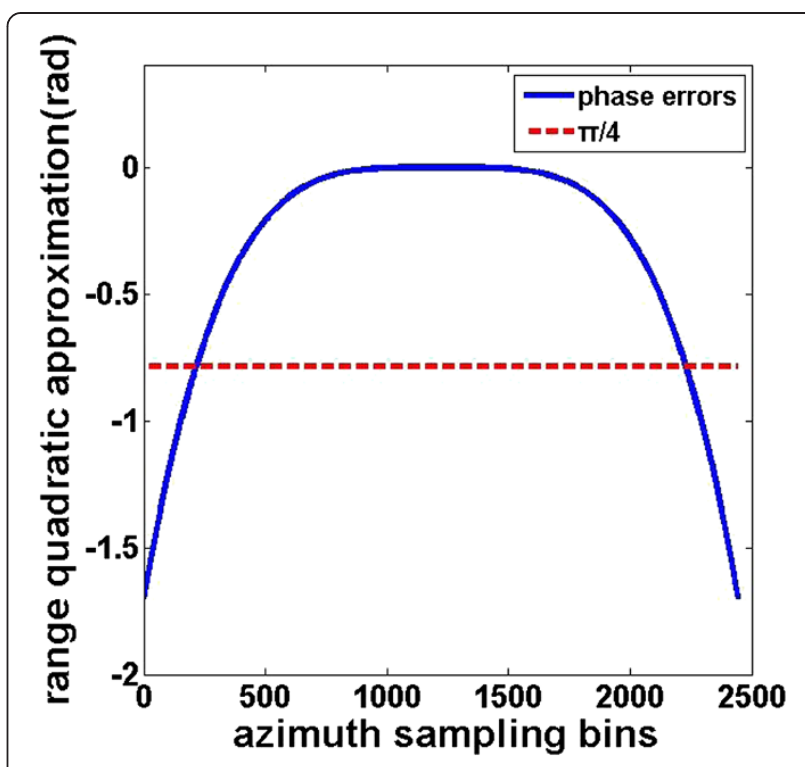

Figure 3 Phase errors for quadratic range approximation.

This term indicates the high-order residue phase which will be compensated in the 2D frequency domain.

Therefore, according to the above analysis, it is possible to acquire the thinking of the modified Omega-K method. Based on the 2D spectrum expression of the SAR echo signal shown in (15) and (16), first the phase of the echo signal is coarsely compensated by performing match filtering for the center point and then the residual range-variant phase is compensated in range Doppler domain.

\subsection{Detailed processing steps}

Based on the above analysis, the full algorithm flow chart is shown in Figure 2.

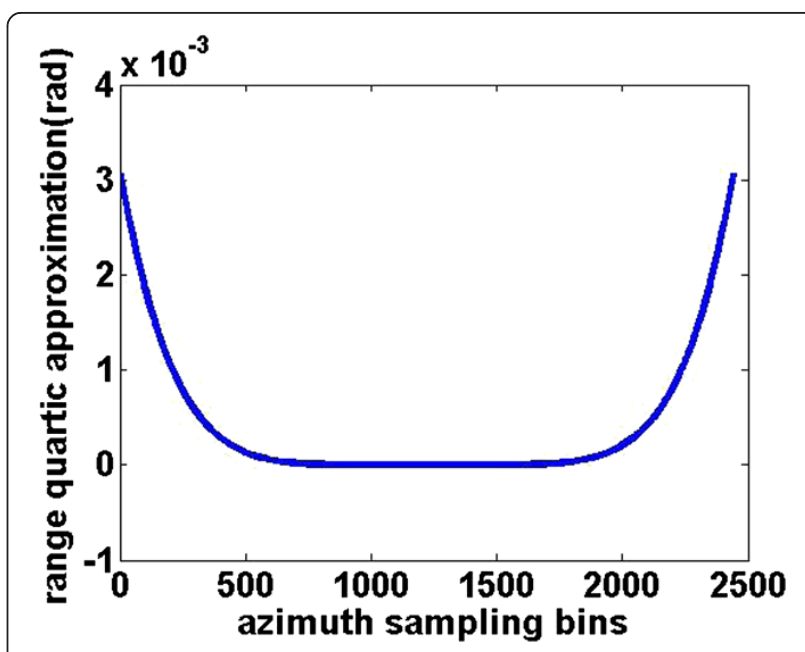

Figure 4 Phase errors for quartic range approximation. 
Table 1 Radar parameters

\begin{tabular}{ll}
\hline Wavelength & $0.03 \mathrm{~m}$ \\
\hline Signal bandwidth & $300 \mathrm{MHz}$ \\
\hline Sampling rate & $500 \mathrm{MHz}$ \\
\hline Pulse width & $10 \mu \mathrm{s}$ \\
\hline Azimuth beam width & $5^{\circ}$ \\
\hline Height & $2000 \mathrm{~m}$ \\
\hline Platform velocity & $100 \mathrm{~m} / \mathrm{s}$ \\
\hline Patch angle & $30^{\circ}$ \\
\hline PRF & $1 \mathrm{kHz}$ \\
\hline Scene center location & $(0,5154.7,0)$ \\
\hline
\end{tabular}

Theoretically, the proposed algorithm can process the data over the full rotation. But in practice, we prefer to divide the data into several parts to alleviate the pressure of the computer memory. There are certain overlaps between two divided block echo data just like the conventional stripmap data processing. It is necessary to clarify that the divided block data should include a whole integration time of the interested target, namely, the interval from the beginning to the end of the illumination of the target. These procedures about azimuth data segmentation, geometric correction, and image mergence are similar to the relative operations represented in [16], readers can refer to them.

The detailed steps to complete imaging are given as follows:

Step 1 Segment the data into blocks in azimuth.

Step 2 2D FFT to transform the echo signal presented in (5) to 2D frequency domain as shown in (15).

Step 3 Compensate the range migration term $\varphi_{\mathrm{rcm}}\left(f_{\eta}\right)$, range modulation term $\varphi_{\mathrm{rg}}\left(f_{\eta}\right)$, residue phase term $\varphi_{\text {res }}$ $\left(f_{\eta}, f_{\tau}\right)$, and SRC term $\varphi_{\text {src }}\left(f_{\eta}\right)$, this can be implemented by multiplying them with their conjugate phase,

$$
H_{1}\left(f_{\tau}, f_{\eta}, R_{0}\right)=\exp \left\{j \varphi\left(f_{\tau}, f_{\eta}\right)\right\}
$$

where

$$
\begin{aligned}
\varphi\left(f_{\tau}, f_{\eta}\right)= & \frac{\pi \cdot c}{4 k_{2}\left(R_{0}\right)}\left(\frac{f_{\eta}}{f_{c}}\right)^{2}-\frac{3 \pi k_{4}\left(R_{0}\right) c^{3}}{64 k_{2}^{4}\left(R_{0}\right)}\left(\frac{f_{\eta}}{f_{c}}\right)^{4}-\frac{\pi \cdot c \cdot f_{\eta}^{2}}{4 k_{2}\left(R_{0}\right) f_{c}^{3}} \\
& +\frac{3 \pi k_{4}\left(R_{0}\right) c^{3} f_{\eta}^{4}}{32 k_{2}^{4}\left(R_{0}\right) f_{c}^{5}}+\frac{\pi \cdot c \cdot f_{\eta}^{2}}{4 k_{2}\left(R_{0}\right)} \frac{f_{\tau}^{3}}{f_{c}^{3}\left(f_{c}+f_{\tau}\right)} \\
& +\frac{\pi k_{4}\left(R_{0}\right) c^{3} f_{\eta}^{4}}{64 k_{2}^{4}\left(R_{0}\right)}\left\{\begin{array}{c}
1 /\left(f_{c}+f_{\tau}\right)-1 / f_{c}^{3} \\
+3 f_{\tau} / f_{c}^{4}-6 f_{\tau}^{2} / f_{c}^{5}
\end{array}\right\}
\end{aligned}
$$

Step 4 Range IFFT to convert the signal to the range Doppler domain and then multiply it with the azimuth match filtering function to finish azimuth compression. The azimuth match filtering function is presented as follows:

$$
H_{2}\left(f_{\eta}, R_{\mathrm{cen}}\right)=\exp \left(-j \frac{\pi \cdot c \cdot f_{\eta}^{2}}{4 k_{2}\left(R_{\mathrm{cen}}\right) f_{c}}+j \frac{\pi \cdot k_{4}\left(R_{\mathrm{cen}}\right) \cdot c^{3} f_{\eta}^{4}}{64 k_{2}^{4}\left(R_{\mathrm{cen}}\right) \cdot f_{c}^{3}}\right)
$$

Step 5 Perform azimuth IFFT to obtain a well-focused SAR image for each block.

Step 6 Merge all the block sub-images into a whole image after operating geometric correction.

\subsection{Theoretical resolution}

For CTSSAR, the analytical expression of range theoretical resolution is the same as that of the conventional rectilinear stripmap SAR, namely, $\rho_{r}=\frac{c}{2 B}$, where $B$ is the bandwidth. However, due to the impact of the circular trajectory, the azimuth theoretical resolution is different from that in the conventional stripmap mode. Assuming that there are two point targets with azimuth angle difference $\Delta \theta$, the Doppler difference of the two targets can be expressed in the following equation based on the range equation (1).

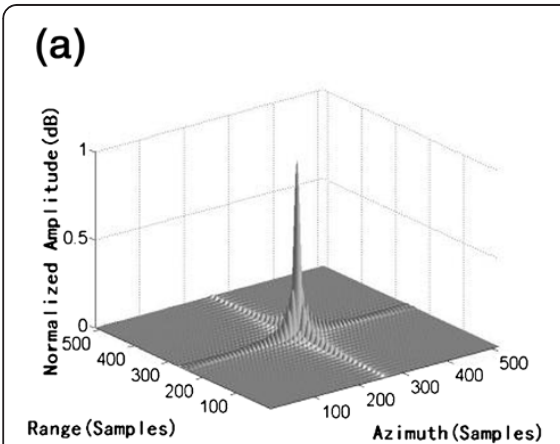

(b)

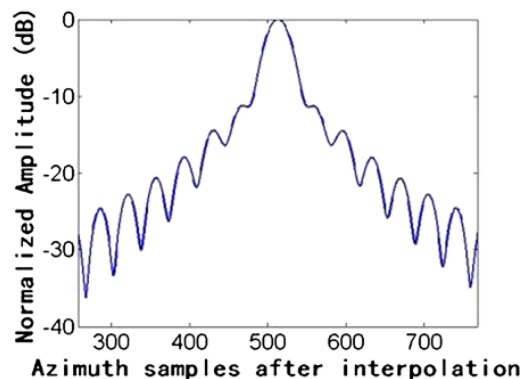

(c)

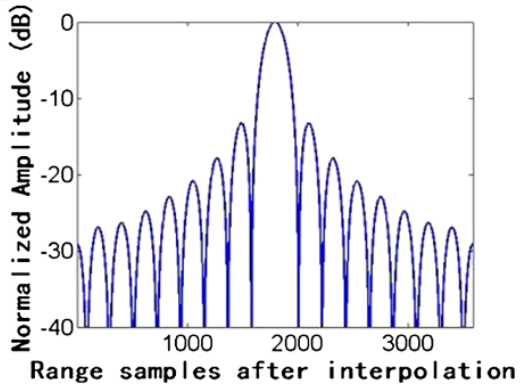

Figure $\mathbf{5}$ Center point imaging result using quadratic approximation method. (a) 2D impulse response. (b) Azimuth impulse response. (c) Range impulse response. 


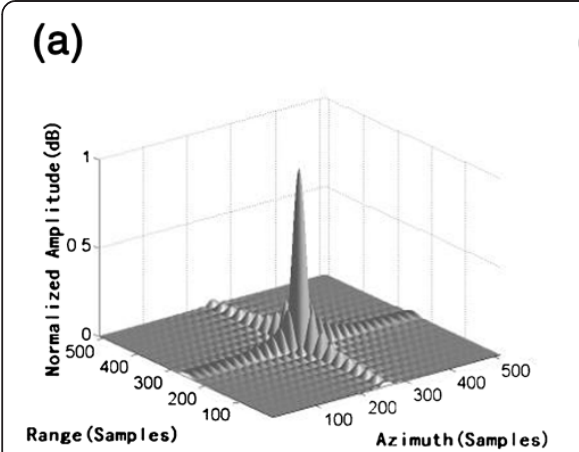

(b)

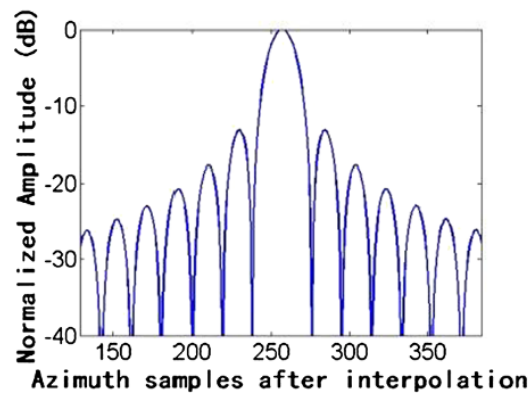

(c)

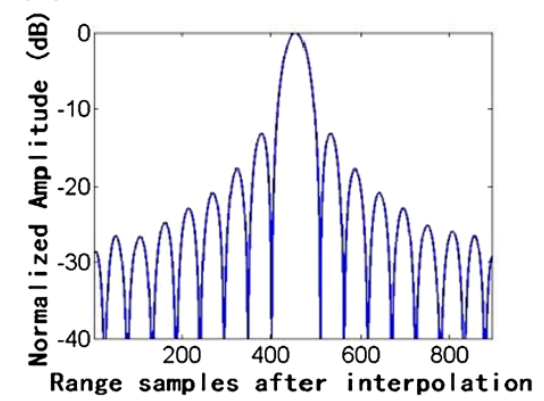

Figure 6 Center point imaging result using the proposed method. (a) 2D impulse response. (b) Azimuth impulse response. (c) Range impulse response.

$$
\Delta f=\frac{2 \omega r_{a} r_{p} \sin \Delta \theta}{\lambda R_{\text {cen }}}
$$

Let the azimuth integration time be $T_{d}$ and the number of it $N$. We suppose that the interval of the two selected point targets is the system minimum distinguishable unit. Then

$$
\frac{f_{\mathrm{PRF}}}{N}=\frac{2 \omega r_{a} r_{p} \Delta \theta}{\lambda R_{\text {cen }}},
$$

where $f_{\mathrm{PRF}}$ denotes the pulse repetition frequency. From (27), the analytical expression for the azimuth theoretical resolution in CTSSAR can be obtained.

$$
\rho_{a}=r_{p} \Delta \theta=\frac{\lambda R_{\mathrm{cen}} f_{\mathrm{PRF}}}{2 N \omega r_{a}}=\frac{\lambda R_{\mathrm{cen}}}{2 v T_{d}}
$$

Here, we use the expression of Doppler FM rate for CTSSAR.

$$
k_{a}=\frac{2 v v_{p}}{\lambda R_{\text {cen }}}
$$

Hence, the expression of azimuth theoretical resolution is obtained, that is, $\rho_{a}=\frac{\lambda R_{\text {cen }}}{2 v T_{d}}$. In addition, relational analysis can be found in [16] and readers can refer to it.

\section{Simulation results}

In SAR imaging, the negligible high-order term error should satisfy the criterion that the maximum double range phase error is less than $\pi / 4$ rad for the range approximation errors [19]. Herein, it is necessary to select an appropriate approximated order. On one side, if the approximation order is too small, the precision will not be large enough to focus the image data. On the other side, too large an approximation order will surely bring certain extra computation, but it will not be the main computational consumption in the practical use for the reason that it is too small compared with the primary computational operation and it can be precalculated and set in the RAM. Nevertheless, more important is that if higher-order range approximation is adopted, the derivation of the 2D spectrum will become very complex and difficult when using MSR [17], especially for the case that the order is larger than fourth-order, the condition under which it is very complicated for calculating the $2 \mathrm{D}$ spectrum. In addition, it is widely accepted that the computational complexity of the time domain BP algorithm is
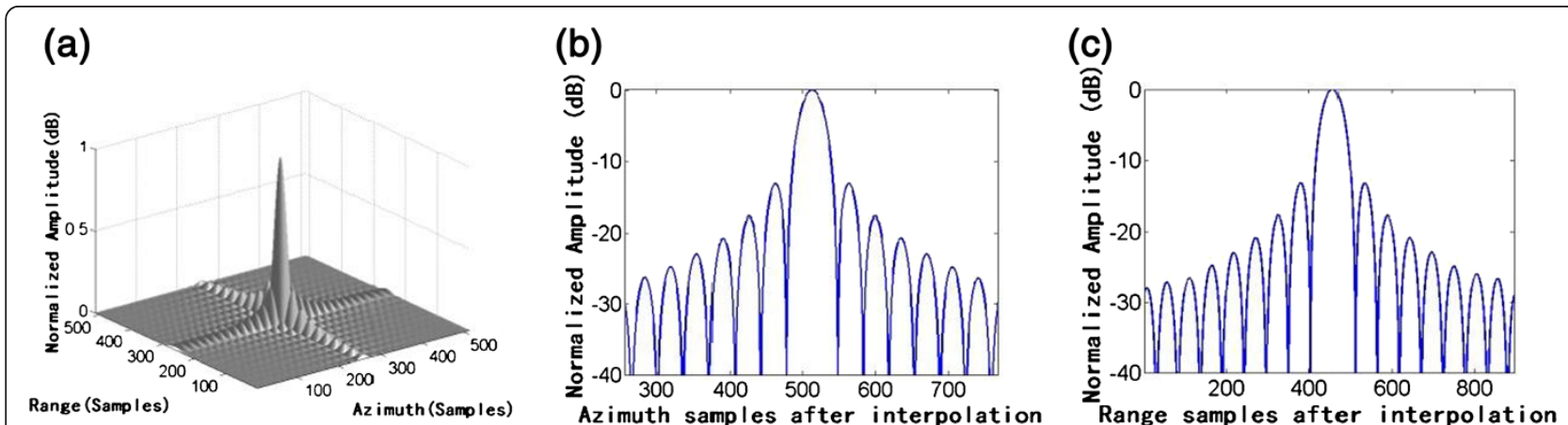

Figure 7 Nearest point imaging result using the proposed method. (a) 2D impulse response. (b) Azimuth impulse response. (c) Range impulse response. 


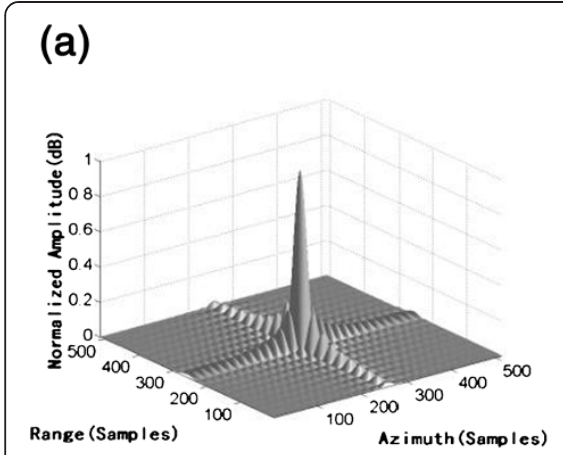

(b)

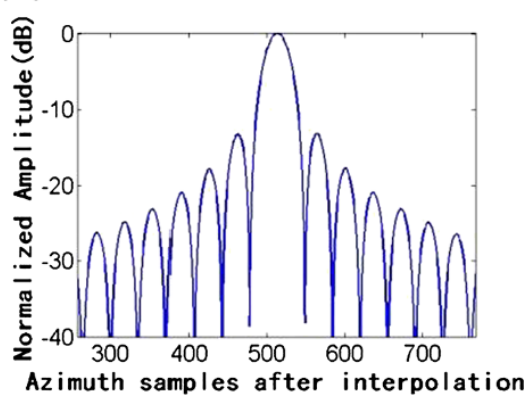

(c)

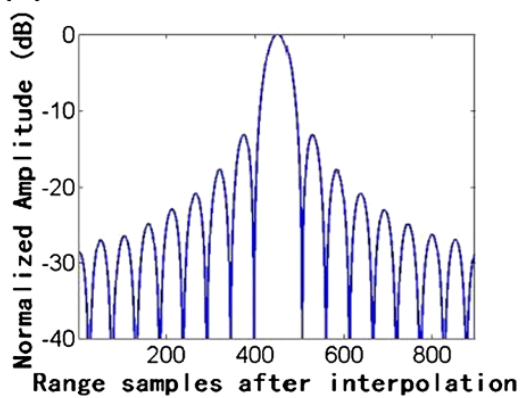

Figure 8 Farthest point imaging result using the proposed method. (a) 2D impulse response. (b) Azimuth impulse response. (c) Range impulse response.

$O\left(N^{3}\right)$ [15]. Taking the complex multiplication into consideration, as illustrated in [19], the number of floating point operations (FLOPs) is used here to estimate the computation load of the algorithm. An FFT or IFFT of length $N$ requires $5 N \log _{2}(N)$ FLOPs. A complex phase multiplication requires six FLOPs. It is assumed that $N_{a}$ is the number of input lines (azimuth samples); $N_{r}$ is the number of input range samples per line. Thus, according to the algorithm flowchart, there are four FFT (or IFFT) operations and two complex multiplication operations:

$$
\begin{aligned}
N_{\text {total }}= & 5 N_{r} N_{a} \log _{2}\left(N_{r}\right) \times 3+6 N_{r} N_{a} \times 2 \\
& +5 N_{r} N_{a} \log _{2}\left(N_{a}\right)
\end{aligned}
$$

Therefore, let $N_{r}=N_{a}=N$, the computational complexity of our algorithm is $O\left(N^{2} \log _{2}(N)\right)$, which is a tremendous improvement for the computational efficiency. In the following simulation, corresponding computational processing time experiments will be carried out with related analyses. Now let us discuss how large the order is needed to satisfy the request of imaging quality.

Here, we choose a series of typical parameters. Assume that the parameters of the flight platform are $H_{c}=2000 \mathrm{~m}, r_{a}=4000 \mathrm{~m}, r_{p}=5154.7 \mathrm{~m}, v=100 \mathrm{~m} / \mathrm{s}$, and the wavelength $\lambda=0.03 \mathrm{~m}$, now compute the quadratic approximation errors and the quartic approximation errors in one synthetic aperture, as shown in Figures 3 and 4 , respectively. The dashed line denotes the location when the phase error is $\pi / 4 \mathrm{rad}$, and the solid line indicates the phase errors introduced by different range approximations in the integrated synthetic aperture time.
Besides, the cubic approximation errors are not considered here for the fact that the odd terms are all zero. It can be seen clearly from the figure that the maximum value of the quadratic approximation errors is about 1.7 $\mathrm{rad}$, larger than $\pi / 4 \mathrm{rad}$ indicated by the dashed line in the figure, which implies that the approximated errors introduced by quadratic approximation cannot be ignored, or the degradation of the image focusing may occur. However, the maximum quadratic approximated errors is about $3 \times 10^{-3} \mathrm{rad}$, far less than $\pi / 4 \mathrm{rad}$, so the dashed line does not noted in the figure. Thus, it is obvious that the expression in (15) is more applicable than the quadratic approximated one.

In order to verify the effectiveness of the proposed algorithm in this article, let us compare our algorithm with the quadratic approximation method in terms of image quality. The simulation parameters are shown in Table 1, there are three target points $P_{n}, P_{m}$, and $P_{f}$ in the scene, and their coordinates are $P_{n}(0,4854.7,0), P_{m}$ $(0,5154.7,0)$, and $P_{f}(0,5454.7,0)$, respectively.

The imaging results of the two algorithms for the same scene center point is shown in Figures 5 and 6 to compare the two algorithms, where (a) is the $2 \mathrm{D}$ impulse response of the target point, (b) is the azimuth impulse response, and (c) is the range impulse response. From the figure, we can see that the quadratic approximation is not able to represent the enough phase variant information and the focusing is not satisfied. In contrast, the algorithm proposed in this article keeps the range up to fourth-order and presents more range-variant information in the $2 \mathrm{D}$ frequency domain. So, it is possible to

\begin{tabular}{|c|c|c|c|c|c|c|c|c|}
\hline \multirow[t]{2}{*}{ Parameter } & \multicolumn{2}{|c|}{ Theoretical one } & \multicolumn{2}{|l|}{$P_{n}$} & \multicolumn{2}{|l|}{$P_{m}$} & \multicolumn{2}{|l|}{$P_{f}$} \\
\hline & Azimuth & $\overline{\text { Range }}$ & Azimuth & $\overline{\text { Range }}$ & Azimuth & $\overline{\text { Range }}$ & Azimuth & Range \\
\hline Resolution (m) & 0.125 & 0.443 & 0.126 & 0.443 & 0.126 & 0.445 & 0.126 & 0.443 \\
\hline PSLR (dB) & -13.26 & & -13.22 & -13.29 & -13.25 & -13.26 & -13.69 & -13.28 \\
\hline ISLR (dB) & -9.8 & & -9.83 & -9.83 & -9.88 & -9.85 & -9.94 & -9.89 \\
\hline
\end{tabular}

Table 2 Image quality parameters using the proposed method 


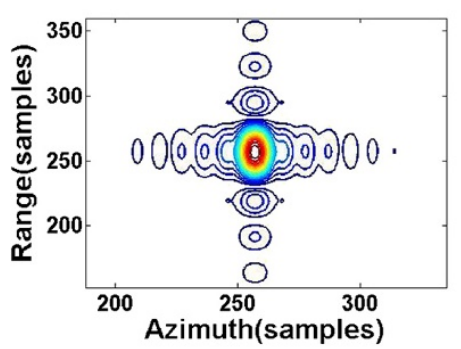

(a) quadratic range model

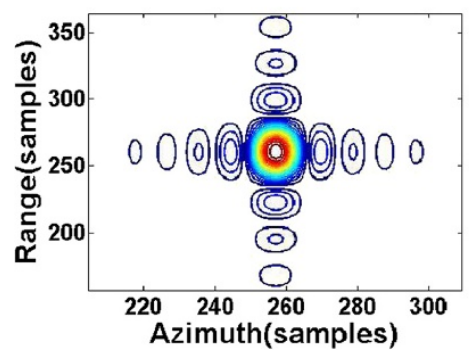

(c) BP

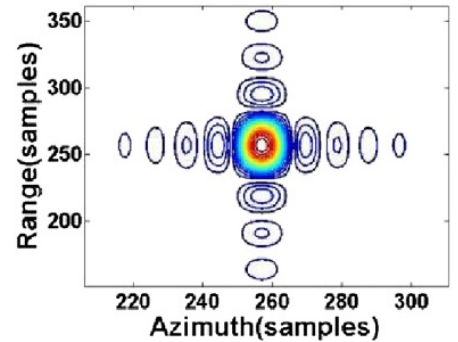

(b) our proposed range model

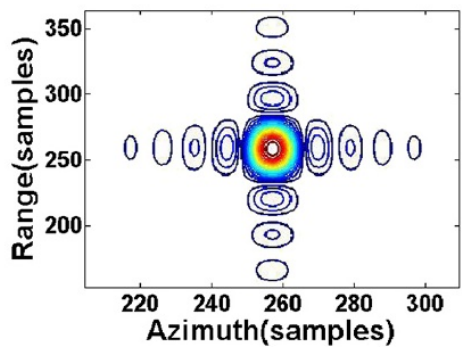

(d) higher order range model

Figure 9 Comparison of different imaging method for a selected point target. (a) Quadratic range model. (b) Our proposed range model. (c) BP. (d) Higher-order range model.

have access to the accurate image with high resolution. Figures 7 and 8 are the imaging results of the nearest point and the farthest point using our method, respectively. It is obvious that not only the center point, but also other points in different range are all well focused, which prove the feasibility of the proposed method successfully.

Table 2 lists the image quality parameters to the three points of our algorithm. Besides, the theoretical range resolution and azimuth resolution are 0.443 and 0.125 $\mathrm{m}$, respectively [16]. It can be seen from Table 2 that each parameter is close to the theoretical one, which indicates satisfactory imaging results and further validates the effectiveness and feasibility.
To further illustrate the superiority of the proposed algorithm over other imaging methods, some imaging results are provided and computational experiments are implemented here. Figure 9 indicates the contour plots of different imaging method for a selected point target $P_{m}$, where Figure 9a delineates the imaging result for the quadratic range approximated algorithm, Figure $9 \mathrm{~b}$ shows the contour of the proposed algorithm based on quartic range approximation, Figure $9 \mathrm{c}$ represents the result of BP algorithm, and Figure 9d is the image for higher-order range approximation (sixth-order is used here). It is obvious that the quadratic approximation is not precise enough to focus the image in CTSSAR and the time domain $\mathrm{BP}$ algorithm seems to be trusted to

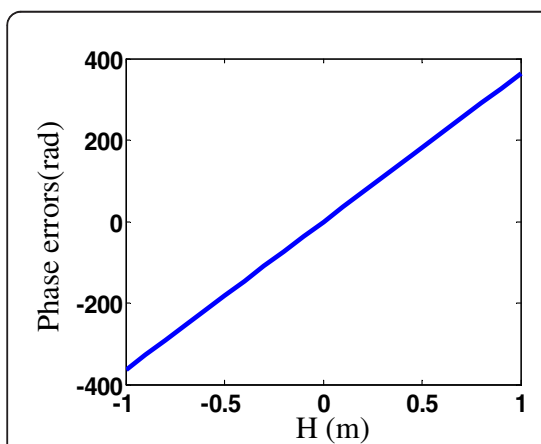

(a) height

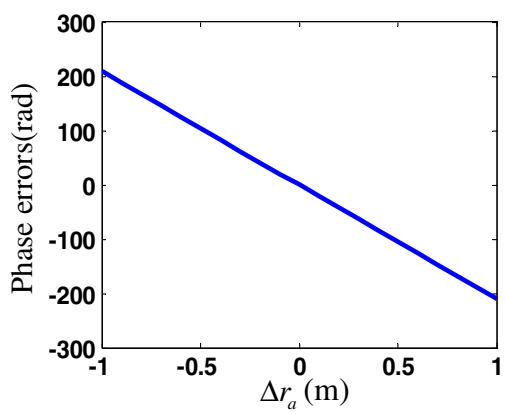

(b) radius

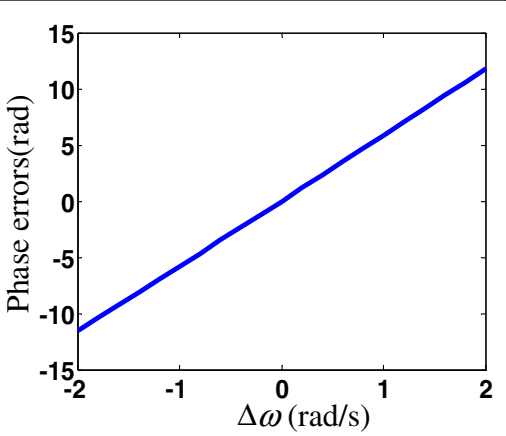

(c) angle velocity

Figure 10 Phase errors caused by each parameter errors. (a) Height. (b) Radius. (c) Angle velocity. 
handle this task perfectly. Unfortunately, too huge the computational load makes it difficult to be applied to the practical engineering use. In contrast, our proposed method can have an access to an ideal result with high computational efficiency. In addition, we can find that the image with higher-order approximation is well focused as well, but we can hardly find any improvement over Figure 9b. So, it confirms that our approximation accuracy is high enough and an extra higher-order approximation appears to be unnecessary in CTSSAR processing. Besides, the higher the approximated order is, the harder the derivation of the 2D spectrum is.

In order to validate the computational efficiency of our algorithm, the computational loads are measured. In our practical experiment, the operation time for the time domain BP algorithm is $14676.80 \mathrm{~s}$, the time for our proposed algorithm is $40.43 \mathrm{~s}$, and the higher-order approximation method (we use sixth-order approximation to simulate here) consumes $48.58 \mathrm{~s}$. Although the quadratic method needs only $26.67 \mathrm{~s}$, we can see obvious defocused phenomenon in the obtained image (shown in Figure 9a). So, we can easily get a conclusion that the BP algorithm is too slow for imaging, the quadratic method cannot reach our imaging standard, and the higher-order method needs a little more time compared with the proposed method (though we can optimize the procedure in the practical use as mentioned before) without any improvement for the imaging. Moreover, the higher-order approximation will bring huge calculating obstacles for the derivation of the 2D spectrum using the MSR. Therefore, after comparison with all kinds of imaging method, it is apparent that the proposed algorithm is a rational, wise choice for CTSSAR.

In practice, the platform cannot always move along an ideal circular path. The trajectory is influenced by many factors. And the sensitivity to the motion errors is analyzed in the following. Three variables of the platform, namely, the height $H_{c}$, the flying radius $r_{a}$, and the angle velocity $\omega$, are selected to carry out the experiment. The simulation experiment results are denoted in Figure 10, indicating the phase errors varying with each parameter errors. In addition, in SAR imaging processing, the maximum phase error for range that will not result in defocus of the image is $\pi / 4 \mathrm{rad}$ [19]. It can be seen from the figures that the phase errors are relatively susceptible to the variations of the parameters. The noticeable degradation will take place when the radius error $\Delta r_{a} \geq 3.75 \times$ $10^{-3} \mathrm{~m}$, the height error $\Delta h \geq 2.165 \times 10^{-3} \mathrm{~m}$, or the angle velocity error $\Delta \omega \geq 0.1346 \mathrm{rad} / \mathrm{m}$. Thus, to compensate for these motion errors, the platform should be equipped with a global positioning system and high accurate inertial navigation system (INS), and more autofocusing techniques are required to be introduced to accomplish fine motion compensation lest the case that the INS cannot meet the request of the motion compensation. Furthermore, if the moving path is too far away from ideal and the trajectory is too complex to make the frequencydomain algorithm being available, the time-domain algorithm (such as BP method) may be an excellent choice.

\section{Conclusions}

In this article, the CTSSAR imaging geometry model is established first, and the imaging difficulties and obstacles are unveiled. Then the point target range function and its 2D frequency spectrum are deduced by MSR, the problem of choosing the approximation order is solved through the analysis of the approximated phase errors and it is verified by related experiments. Based on the derived spectrum, the corresponding modified Omega-K method is developed. The high-order term introduced from the circular track is compensated at the beginning of the algorithm. Range compression and RCMC are then conducted in 2D domain followed with azimuth compression to obtain a sub-image, the ultimate image is formed by merging all the sub-images together. Compared with the quadratic approximation method, the proposed method has more accuracy. At the end of the article, the feasibility and efficiency of the proposed approach are validated with the simulation experiments.

\section{Competing interests}

The authors declare that they have no competing interests.

\section{Acknowledgments}

The authors would like to thank the anonymous reviewers for their careful reading of this article and for their detailed and constructive comments that have improved the presentation of this article. This study was supported in part by the National Natural Science Foundation of China under grant 61222108 and in part by the "973" program of China under grant 2010 CB731903.

Received: 28 June 2012 Accepted: 4 March 2013 Published: 28 March 2013

\section{References}

1. RK Raney, H Runge, R Bamler, IG Cumming, FH Wong, Precision SAR processing using chirp scaling. IEEE Trans. Geosci. Remote Sens. 32, 786-799 (1994)

2. N Gebert, G Krieger, Azimuth phase center adaptation on transmit for highresolution wide-swath SAR imaging. IEEE Geosci. Remote Sens. Lett. 6, 782-786 (2009)

3. C Cafforio, C Prati, F Rocca, SAR data focusing using seismic migration techniques. IEEE Trans. Aeorsp. Electron. Syst. 27, 194-207 (1991)

4. M Soumekh, Synthetic Aperture Radar Signal Processing With Matlab Algorithms (Wiley, New York, 1999)

5. K Knaell, Three-dimensional SAR from curvilinear apertures. Proceedings of IEEE Radar Conference, Carderock Div 2230, 220-225 (1996). NSWC, Bethesda, MD, USA

6. D Falconer, G Moussally, Tomographic imaging of radar data gathered on a circular flight path about a three-dimensional target zone. Proceedings of SPIE, Aerosp, Syrup 2487:2-12 (1995). Orlando, FL

7. A Ishimaru, T Chan, Y Kuga, An imaging technique using confocal circular synthetic aperture radar. IEEE Trans. Geosci. Remote Sens. 36, 1524-1530 (1998)

8. M Bara, L Sagues, F Paniahua, A Broquetas, X Fabregas, High-speed focusing algorithm for circular synthetic aperture radar. Electron. Lett. $36,828-830$ (2000) 
9. H Cantalloube, EC Koeniquer, Airborne SAR imaging along a circular trajectory, in (Proceedings of EUSAR, Dresden, Germany, 2006), pp. 16-19

10. H Cantalloube, EC Koeniquer, H Oriot, High resolution SAR imaging along circular trajectories (Proceedings of IGARSS, Spain, Barcelona, 2007), pp. 850-853

11. M Soumekh, Reconnaissance with slant plane circular SAR imaging. IEEE Trans. Image Process. 5, 1252-1265 (1996)

12. M Ferrara, JA Jackson, C Austin, Enhancement of multi-pass 3D circular SAR images using sparse reconstruction techniques, in. Algorithms for Synthetic Aperture Radar Imagery XVI, Proceedings of SPIE 7337, 733702 (2009). Orlando, FL

13. M Pinheiro, P Prats, R Scheiber, M Nannini, A Reigber, Tomographic 3D reconstruction from airborne circular SAR. Proceedings of IGARSS 3, III21-III24 (2009). Cape Town, South Africa

14. O Ponce, P Prats, MR Cassola, R Scheiber, A Reigber, Processing of circular SAR trajectories with fast factorized back-projection, in Proceedings of IGARSS (Proceedings of IGARSS, Vancouver, Canada, 2011), pp. 3692-3695

15. $Y$ Lin, $W$ Hong, WX Tan, YR Wu, Extension of range migration algorithm to squint circular SAR imaging. IEEE Geosci. Remote Sens. Lett. 8, 651-655 (2011)

16. B Sun, YQ Zhou, J Chen, CS Li, Operation mode of circular trace scanning SAR for wide observation. J. Electron. Inf. Technol. 30, 2805-2808 (2008)

17. YL Neo, FH Wong, IG Cumming, A two-dimensional spectrum for bistatic SAR processing using series reversion. IEEE Geosci. Remote Sens. Lett. 4, 93-96 (2007)

18. YL Neo, FH Wong, IG Cumming, Processing of azimuth-invariant bistatic SAR data using the range Doppler algorithm. IEEE Trans. Geosci. Remote Sens. 46, 14-21 (2008)

19. IG Cumming, HW Frank, Digital Processing of Synthetic Aperture Radar Data: Algorithms and Implementation (Artech House, Norwood, MA, 2005)

doi:10.1186/1687-6180-2013-64

Cite this article as: Liao et al:: A novel modified Omega-K algorithm for circular trajectory scanning SAR imaging using series reversion. EURASIP Journal on Advances in Signal Processing 2013 2013:64.

\section{Submit your manuscript to a SpringerOpen ${ }^{\circ}$ journal and benefit from:}

- Convenient online submission

- Rigorous peer review

- Immediate publication on acceptance

- Open access: articles freely available online

- High visibility within the field

- Retaining the copyright to your article 